\title{
Stir Bar Sorption Extraction (SBSE) and Its Application for Analysis of Organic Compounds in Aqueous Samples: A Review
}

\author{
Mesfin Medihin Ododo \\ Department of Chemistry, Wolaita Sodo University, Wolaita Sodo, Ethiopia
}

\begin{abstract}
Sample preparation is one of the crucial steps in any chemical analysis because most samples are not ready to introduce directly into analytical instruments. This step is very important to eliminate interferences, isolate and preconcentration of analytes of interest from the matrix and makes them more suitable for separation and detection. The extraction of analytes from aqueous matrices can be traditional and nontraditional techniques. Miniaturized techniques are simple, solventless or solvent-reduced techniques allowing the extraction and concentration in a single step on a micro scale approach. Stir Bar Sorptive Extraction (SBSE) is a relatively new solventless sample preparation method for the extraction and concentration of organic compounds from aqueous matrices of environmental, food and biological samples. In SBSE, a stir bar coated with Polydimethylsiloxane (PDMS) phase is added to a vial containing the sample. The sample is then stirred until analytes partition equilibrium time reached with sorbent. The extracts can be introduced quantitatively into the analytical system by thermal or liquid desorption. The efficiency of extraction of SBSE in terms of the amount extracted and the equilibrium is affected by extraction time, stirring speed, temperature of the sample, $\mathrm{pH}$, salting and sample volume. The most important limitation of SBSE is the only commercial availability of PDMS coating material exists at present. Besides, due to the non-polar character of PDMS, SBSE technique is mainly applied to extract non-polar and weakly polar compounds but failed in extraction of strongly polar compounds.
\end{abstract}

Keywords: Stir Bar Sorptive Extraction, Polydimethylsiloxane, Sample Preparation, Organic Compounds DOI: $10.7176 / \mathrm{CMR} / 13-1-01$

Publication date: January $31^{\text {st }} 2021$

\section{Introduction}

Sample preparation is a process required for the transformation of the analytes of interest from sample matrix into a form more suitable for introduction into the analytical instrument (Baltussen, 2000). It is very important to extract, isolate, and concentrate the analytes of interest from complex matrices into a suitable solvent. Thus, it has a direct impact on accuracy, precision, quantification limits and the rate-determining step of the analytical methods, especially when trace analysis is the main purpose (Feilden, 2011).

The extraction of analytes from aqueous matrices can be traditional and nontraditional techniques (Jochmann, 2006). The most common traditional and the most widely used technique is liquid-liquid extraction (LLE). However, it is tedious, time-consuming, difficult to automate and environmentally unfriendly due to large amounts of organic solvents are required. Solid-phase extraction (SPE) was introduced as an alternative extraction method. Comparing to LLE, this method reduces high solvent consumption. The major drawbacks of SPE are the large sample volumes requirement and limited enrichment factor (Ochiai et al., 2008).

In order to achieve adequate detection limits, it is often necessary to either perform concentration to a small volume or use large volume injection. For this reason, miniaturized methods (microextraction approaches), which are simple, solventless or solvent-reduced techniques allowing the extraction and concentration in a single step on a micro scale approach were developed. After Lord and Pawliszyn, microextraction is defined as a technique where the volume of the extraction phase is very small in relation to the sample volume (Jochmann, 2006). Solid Phase Microextraction (SPME) and Stir Bar Sorptive Extraction (SBSE) are typical examples of miniaturization in sample preparation techniques (Lokhnauth, 2005). SPME is the most prominent and widely used solventless microextraction technique for organic compounds in aqueous samples. However, the applicability of SPMS is occasionally limited by the small amount of coating material that is present on the fiber (Jochmann, 2006). SBSE was developed to overcome the limited extraction capacity of SPME fibers (Tan and Chai, 2011).

SBSE is a relatively new solventless sample preparation method for the extraction and concentration of organic compounds from aqueous matrices (Lokhnauth, 2005). In SBSE, a stir bar coated with Polydimethylsiloxane (PDMS) phase is added to a vial containing the sample. The sample is then stirred until analytes partition equilibrium time reached with sorbent. After the extraction, the analytes can be introduced quantitatively into the analytical system by thermal or liquid desorption (Kassem, 2011). SBSE is widely applied in analysis of trace organic compounds in aqueous matrices of environmental, food and biological samples. However, its important limitation is only commercial availability of PDMS coating material exists at present (Tan and Chai, 2011). Besides, due to the non-polar character of PDMS, SBSE technique is mainly applied to extract non-polar and weakly polar compounds but failed in the extraction of strongly polar compounds (Huang et al., 2009). 
The objectives of this review were to address the working principles and instrumental set-up of SBSE technique, to compare SBSE with other analytical technique particularly SPME and to address its application for analysis of organic compounds in environmental, biological and food aqueous samples.

\section{Working Principle}

\subsection{Polydimethylsiloxane (PDMS) Coated Stir Bars}

PDMS coated stir bars are only commercially available materials used for SBSE method under the trade name Twister ${ }^{\mathrm{TM}}$ from Gerstel GmbH (Mülheim an der Ruhr, Germany) (Baltussen, 2000; Kole et al., 2011). PDMS stir bars (Twister ${ }^{\mathrm{TM}}$ ) are commercially available from Gerstle in two configurations, both coated a with 1 mm layer of PDMS: $10 \mathrm{~mm} \mathrm{~L} \mathrm{x} 3.2 \mathrm{~mm}$ o.d and $40 \mathrm{~mm} \mathrm{~L}$ x $3.2 \mathrm{~mm}$ o.d (Lokhnauth, 2005). Typically, the $10 \mathrm{~mm}$ stir bars are used for 1-50 $\mathrm{mL}$ sample volumes, and the $40 \mathrm{~mm}$ stir bars are used for sample volumes up to $250 \mathrm{~mL}$ sample volumes (Baltussen, 2000). These stir bars have three essential parts as shown in Figure 1. The first and innermost part is a magnetic stirring rod, which is necessary for transferring the rotating movement of a stirring plate to the sample liquid. The second part of the stir bar is a thin glass jacket that covers the magnetic stirring rod. The third and outermost part is the layer of PDMS sorbent into which the analytes extraction occurs. The glass layer is essential in the construction of a high quality stir bar. It effectively prevents decomposition of the PDMS layer, catalysed by the metal of the magnetic rod (Baltussen, 2000; Lokhnauth, 2005; David et al., 2003).

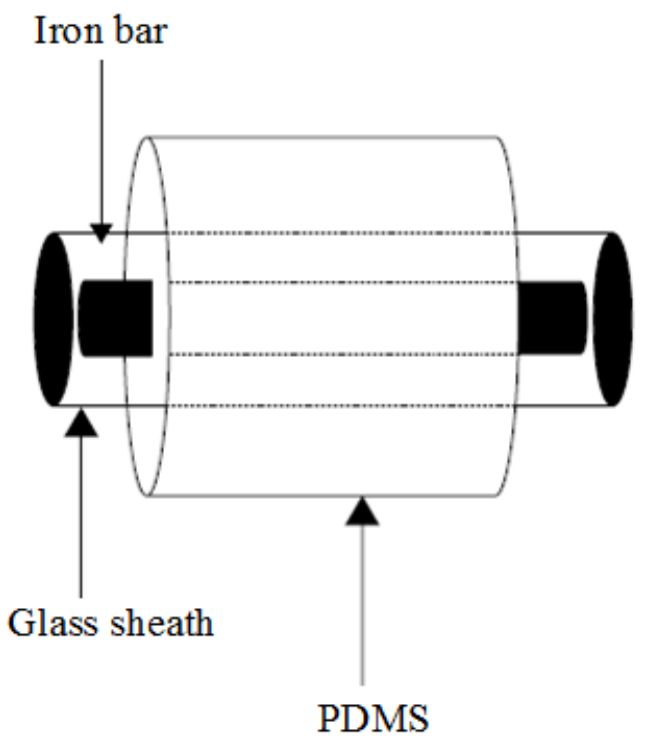

Figure 1. Schematic representation of a PDMS coated stir bar for SBSE application (Mitra, 2003).

Stir bars are used in two application modes for extraction. The first one is the direct immersion of the PDMS stir bar into the sample, and the other one is analyte extraction from headspace above the sample as shown in Figure $2 \mathrm{a}$ and $\mathrm{b}$, respectively (Jochmann, 2006).

\subsection{Extraction Procedure}

The stir bar coated with PDMS phase is directly added to a vial containing the sample as shown in Figure 2 a (Kassem, 2011), or special device to hold the stir bar is available from headspace above the sample as shown in Figure $2 \mathrm{~b}$ (Kole et al., 2011). Then, it is stirred until analytes partition equilibrium time reached with sorbent. A magnetic stirrer is used to set the stir bar in rotation, and this enhances the mass transport in the system and thus increases the extraction rate (Pettersson, 2004). As shown in Figure 2 b, headspace bars consist of a glass rod of ca. $5 \mathrm{~cm}$ length with PDMS tubing over the last $\mathrm{cm}$. These rods are mounted in the screw caps of headspace vials (Jochmann, 2006).

As the name suggests, SBSE is based on sorptive extraction, and is by nature an equilibrium technique (Kole et al., 2011). The amount of analyte extracted from the aqueous phase is controlled by the partitioning coefficient of solutes between the polymer coating and sample matrices $\left(K_{P D M S / W}\right)$ for direct immersion. The headspace extraction is dependent on the distribution of the analytes between the liquid and gaseous headspace, and on the respective PDMS/gas partition coefficients (Tredoux, 2008) and the phase ratio between polymer coating and sample volume $(\beta)$ (Lanuza, 2010). 


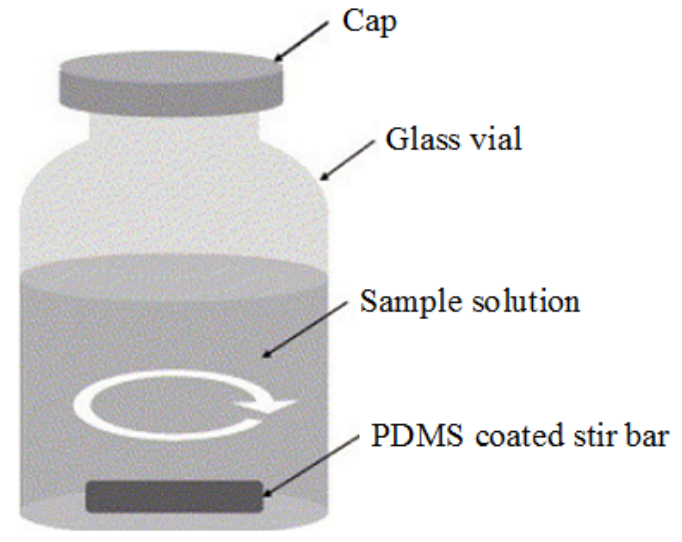

a)

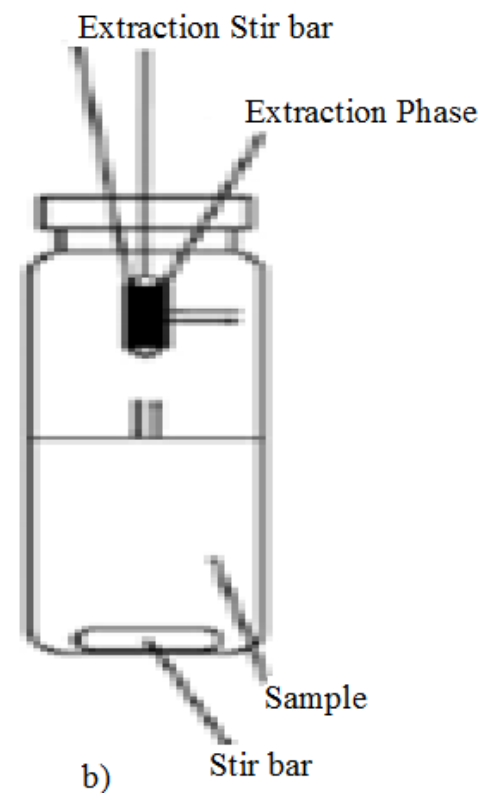

b) Stir bar

Figure 2. Schematic Diagram of SBSE setup for a) direct immersion (Tan and Chai, 2011) and b) headspace (Jochmann, 2006)

The distribution coefficient between PDMS and water $\left(K_{P D M S / W}\right)$ is defined as the ratio between the concentrations of a solute in PDMS phase $\left(C_{P D M S}\right)$ over the concentration in water $\left(C_{W}\right)$ at equilibrium (Kassem, 2011).

$$
K_{P D M S / W}=\frac{C_{P D M S}}{C_{W}}=\frac{m_{P D M S}}{m_{W}} \times \frac{V_{W}}{V_{P D M S}}
$$

Where, $m_{P D M S}$ and $m_{W}$ is the mass of analyte in the PDMS and the water phase respectively, and $V_{P D M S}$ and $V_{W}$ is the volume of the PDMS and water phases respectively. This ratio is equal to ratio of the $m_{P D M S}$ over the $m_{W}$ times the phase ratio $\beta$, where $\beta=\frac{V_{W}}{V_{P D M S}}$ (Kassem, 2011). Thus, equation (1) can be re-written as:

$$
K_{P D M S / W}=\beta \frac{m_{P D M S}}{m_{W}}
$$

Recent studies have correlated this partition coefficient with the octanol-water partition coefficients $\left(K_{O / W}\right)$ which gives a good estimation on the ability of the coating to extract an analyte (Kole et al., 2011; David et al., 2003).

If the approximation is made, $K_{P D M S / W}$ is proportional to the $K_{O / W}$, it can be stated that (Lokhnauth, 2005):

$$
K_{P D M S} \approx K_{O / W}=\beta \frac{m_{P D M S}}{m_{W}}
$$

The theoretical recovery or extraction efficiency can therefore be calculated based on the distribution constant of the analyte and a known phase ratio, which is expressed as the ratio of the extracted amount of solute $\left(m_{P D M S}\right)$ over the total amount of solute originally present in the water $\left(m_{O}=m_{W}+m_{P D M S} \Rightarrow m_{W}=m_{O}-m_{P D M S}\right)$. And equation (3) can be re-arranged as (David et al., 2003):

$$
\frac{K_{O / W}}{\beta}=\frac{m_{P D M S}}{m_{W}}=\frac{m_{P D M S}}{m_{O}-m_{P D M S}}
$$

Equation (4) can be re-arranged in such a way as to determine the extraction efficiency or recovery from the water samples as follows: 


$$
\frac{m_{P D M S}}{m_{O}}=\frac{\left(\frac{K_{O / W}}{\beta}\right)}{1+\left(\frac{K_{O / W}}{\beta}\right)}
$$

Thus, theoretical recovery $(\%)=\frac{m_{P D M S}}{m_{O}} \times 100 \%=\frac{\left(\frac{K_{O / W}}{\beta}\right)}{1+\left(\frac{K_{O / W}}{\beta}\right)} \times 100 \% \ldots \ldots \ldots \ldots \ldots \ldots \ldots \ldots . . . .6$

Using above equation (6), analysts can calculate the extraction efficiencies for a solute with known partition coefficient and a given phase ratio. It is also clear that the extraction efficiency increases with increasing $K_{P D M S / W}$. Since $K_{P D M S / W}$ is similar to the $K_{O / W}$, extraction efficiency on PDMS, in general, decreases with increasing polarity. Besides, $K_{P D M S / W}$ factor and phase ratio $(\beta)$ also are important. The higher PDMS amount and the lower $\beta$ value results higher extraction efficiency (Kassem, 2011).

Figure 3 illustrates the recovery of extraction as a function of the $\frac{K_{O / W}}{\beta}$. At $\frac{K_{O / W}}{\beta}=1$ the recovery is $50 \%$. At low $\frac{K_{O / W}}{\beta}$ the value of recovery is approximately equal to the $\frac{K_{O / W}}{\beta}$ while at $\frac{K_{O / W}}{\beta}$ value higher than 5 extractions is essentially quantitative (Baltussen, 2000; Tredoux, 2008). The theoretical recoveries can be calculated for a given sample volume, selected stir-bar dimensions and a solute using the KowWIN software program (Syracuse Research Corp., Syracuse, New York, USA), which is based upon a $\log K_{O / W}$ calculator (Kassem, 2011).

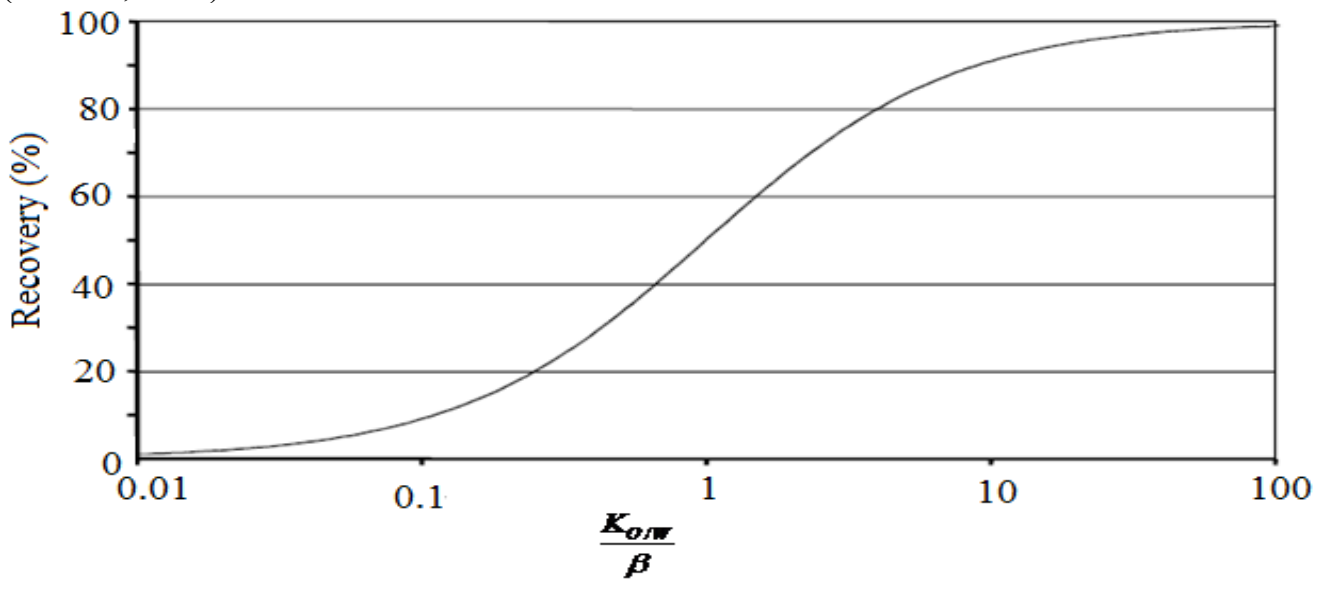

Figure 3. Recovery as a function of the ratio of octanol-water partition coefficient and phase ratio $\left(\frac{K_{O / W}}{\beta}\right)$ (Baltussen, 2000)

\subsection{Optimization of the Parameters that Affect SBSE Efficiency}

The efficiency of extraction of SBSE in terms of the amount extracted and the equilibrium is affected by extraction time, stirring speed, temperature of the sample, $\mathrm{pH}$, salting and sample volume. These factors can be optimized individually or one can use a design of experiments approach (Kole et al., 2011).

\subsubsection{Extraction Time}

The length of extraction time is necessary to reach equilibrium in SBSE. The extraction time profile is obtained by first preparing samples and extracting them for progressively longer period of time and then determines the response for each point time (Lokhnauth, 2005). The extraction time is controlled kinetically; determined by sample volume, stirring speed and stir bar dimensions. Optimization of extraction time for a given application is normally accomplished by measuring the analyte recovery as a function of the extraction time. Equilibrium condition is reached when no additional recovery is observed when the extraction time is increased further (David 
et al., 2003). Non-equilibrium conditions can also be used in SBSE when the equilibrium time is excessively long and sensitivity of the technique is not of greatest concern (Lokhnauth, 2005). Comparing to non-equilibrium methods, equilibrium methods tend to be simpler, less expensive and more selective (Mitra, 2003).

\subsubsection{Stirring Rate}

Digitally controlled stirrers are ideal for SBSE, since it is important that all extractions are performed at the setting. During method development, rotation speed studies can be done by carrying out the extraction at various stirring speeds to determine the optimum extraction rate. During stirring, the stir bar should not be moving erratically, but in a stable circular motion to achieve efficient contact and allow rapid mass transfer of analyte into the PDMS. Extraction is efficient when fast rotational speeds are applied with the equilibration time increasingly decrease as the revolutions per minute (rpm) of the stir bar increases (Lokhnauth, 2005).

\subsubsection{Temperature}

Increasing the temperature of the sample, during SBSE, could potentially reduce the equilibrium time by increasing the diffusion coefficients of the analytes. However, increasing the temperature can lower the analyte partition coefficient thereby reducing the amount of analyte transferred from the sample to the stir bar coating. The optimum extraction temperature should be determined experimentally, which is usually accomplished by constructing a temperature profile for the extracted analytes. The optimization of the temperature is used to achieve the desired sensitivity in the shortest possible time (Lokhnauth, 2005).

\subsection{4 pH}

The $\mathrm{pH}$ of the sample is an important factor in SBSE when the analytes of interests are polar compounds, acids, or bases. In SBSE, there is only the nonpolar PDMS coating commercially available, and therefore, it is more important to reduce the polarity of analytes by making $\mathrm{pH}$ adjustments. For these types of analytes, changing the $\mathrm{pH}$ of the sample matrix determines whether the species exist as an associated or dissociated moiety, and there by offering method selectivity in terms of whether or not the analyte gets extracted. For example, in the PDMS coating, as the $\mathrm{pH}$ is lowered, more acidic analyte is present in neutral forms making it more likely to partition into the coating, resulting in higher sensitivity (Lokhnauth, 2005).

\subsubsection{Salting Effect}

Increasing salt concentrations of a sample matrix can increase or decrease the amount of analyte extracted by the stir bar. Due to nonpolarity of PDMS, generally, it is important to first convert the charged analytes into neutral forms. The solubilities of many organic compounds in an aqueous solution decrease in the presence of excess salt. Hence, increase the partition coefficient of analytes between the sample matrix and coating is to add salt to the matrix (Lokhnauth, 2005).

\subsubsection{Sample Volume}

The distribution constant determines minimum sample volume that should be used in an SBSE method. It can be determined experimentally. Typically, $10-50 \mathrm{~mL}$ sample volumes are used for the $10 \mathrm{~mm}$ stir bars, and up to 250 $\mathrm{mL}$ sample volumes are used for the $40 \mathrm{~mm}$ stir bars (Lokhnauth, 2005).

\subsection{Rinse and Dry of PDMS Coated Stir Bar}

After an extraction has been carried out, the stir bar device is manually removed from the sample container using cleaned magnetic tweezers, slightly rinsed with distilled water to remove undesired sample components and finally dried by wiping it with a lint free tissue as shown in Figure 4 (Pettersson, 2004; Lanuza, 2010). Gentle rinsing would not cause any analyte loss; because, the sorbed analytes of interest present in the PDMS phase (Tan and Chai, 2011; David et al., 2003).

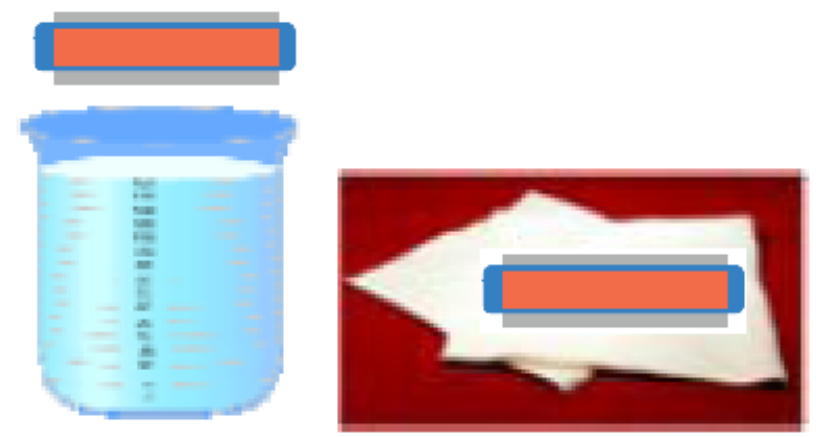

Figure 4. Rinse and drying of PDMS coated stir bar after removing from the sample (Margoum et al., 2008)

\subsection{Desorption Step}

Finally, the analytes must be desorbed either thermally using a designated desorption unit or by extraction with a suitable solvent (Pettersson, 2004). For volatile compounds, the stir bar is introduced in an empty glass thermal 
desorption tube, and then it is placed into a Gerstel Thermal Desorption System (TDS) or Thermal Desorption Unit (TDU) where the analytes are thermally desorbed (Baltussen, 2000). Then it can be on-line transferred to Gas Chromatography (GC) column or GC-Mass Spectrometry (MS) system for further analysis (Corderoet al., 2009). The desorption conditions such as desorption temperature, desorption time and flow rate of the carrier gas are usually optimized during method optimization. The optimum temperature for desorption of analytes from the stir bar is analyte dependent. Depending on the concentration and volatility of the analytes, desorption can be achieved between $150{ }^{\circ} \mathrm{C}$ and $300{ }^{\circ} \mathrm{C}$ in 5 to $15 \mathrm{~min}$ under a $10-50 \mathrm{~mL} / \mathrm{min}$ gas flow. Higher flow rates are more desirable for effective desorption, especially if the analytes or interest are less volatile. To ensure that complete desorption is occurring, carryover studies can be performed by redesorbing the stir bar after the initial run (David et al., 2003).

Alternatively, less volatile, non-volatile or thermally unstable analytes can removed from the stir bars using solvent desorption and injected into a LC or LC-MS (Feilden, 2011). When performing LD, factors such as type of solvent, desorption time, stirring speed and temperature must be optimized to obtain the required sensitivity (Kole et al., 2011). Solubility factors are important in solvent selection because the analyte should preferentially partition from the stir bar coating into the solvent. The most widely used are polar solvents, mainly acetonitrile and less used are methanol, acetone, and ethyl acetate, in which PDMS is not soluble (Corderoet al., 2009). Increasing temperature and stirring rate can significantly improve the desorption efficiency (Lokhnauth, 2005).

After either thermal desorption or LD, the stir bars can be reused. To reuse, the stir bars must be cleaned with suitable solvent mixture for some minutes at specified temperature with magnetic stirring. This step should be repeated at least three times with fresh portions of the solvent mixture. After drying with a lint-free tissue, stir bar must be kept in a vial for the next analysis (Lanuza, 2010). Typically, the lifetime of a single stir bar is approximately 20 to 50 extractions, depending on the matrix (Tan and Chai, 2011).

\section{Instrumentation}

\subsection{For Thermally Desorbing Compounds}

The instrumental set-up consists of a Gerstel TDS, a temperature programmable Cooled Injection System (CIS), a gas chromatograph /or, detector and readout system as shown in Figure 5 (Hoffmann and Bremer, 1994). In this technique, after extraction, the stir bar is removed from the sample, placed in a glass thermal desorption tube (Lanuza, 2010) and then inserted into the TDS which is cooled down to subambient temperatures in order to prevent premature desorption (Hoffmann and Bremer, 1994). TDS uses a programmed temperature vaporizer (PTV) injector which is operated as a cytotrap for cryogenic refocusing of the thermally desorbed analytes (David et al., 2003). After purging the air out of the system, the tube is heated to the desired temperature, while the carrier gas flowing through the tube transfer's the volatiles in split or splitless mode into the pre-cooled CIS, where they are cryofocused (Hoffmann and Bremer, 1994). Liquid nitrogen is used for cooling where temperatures as low as $-15^{\circ} \mathrm{C}$ are used (David et al., 2003). After desorption is finished, the CIS is heated to the desired temperature to allow split or splitless transfer of the trapped compounds to GC-MS system for further analysis.

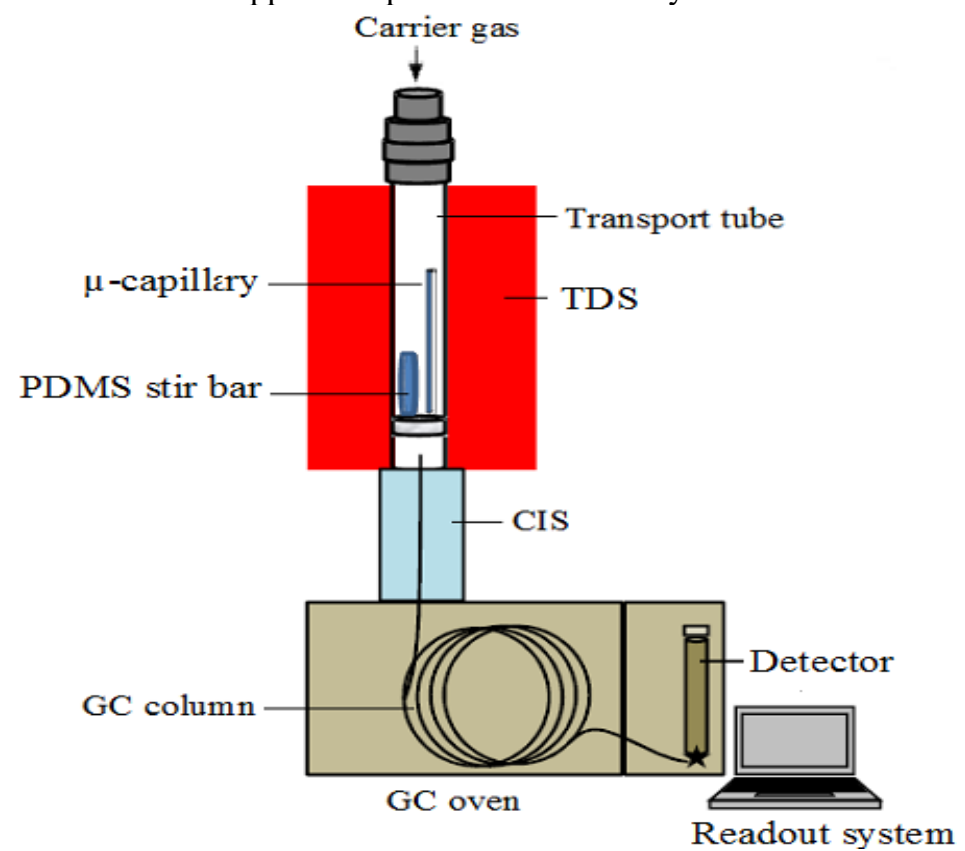

Figure 5. Schematic representation of the SBSE instrumental set-up for thermally desorbing compounds (Skogerson et al., 2011) 


\subsection{For Solvent Desorbing Compounds}

For thermally labile or high molecular weight compounds, the combination of SBSE and LC or LC-MS instrument is useful. Liquid desorption of the extracted analytes is accomplished by placing the stir bar in a vial or an insert containing a small amount of organic solvent, which is suitable for the target analytes. Following sonication (Kole et al., 2011) or magnetic stirring, where the stir bar is used to stir the solvent (Lanuza, 2010), a portion of the solvent is transferred to the LC system. Detection can be accomplished by means detectors such as fluorescence or ultraviolet (UV) or by MS (Stopforth, 2007). The instrumental set-up for solvent desorbing compounds is given in Figure 6.

\section{After drying}

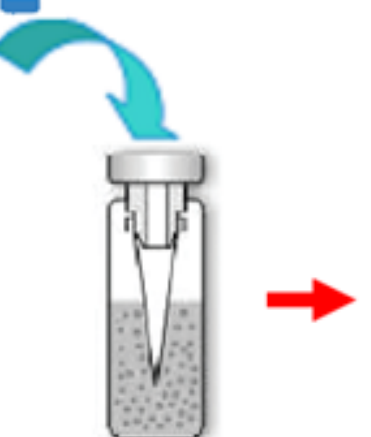

Vial contains organic solvent
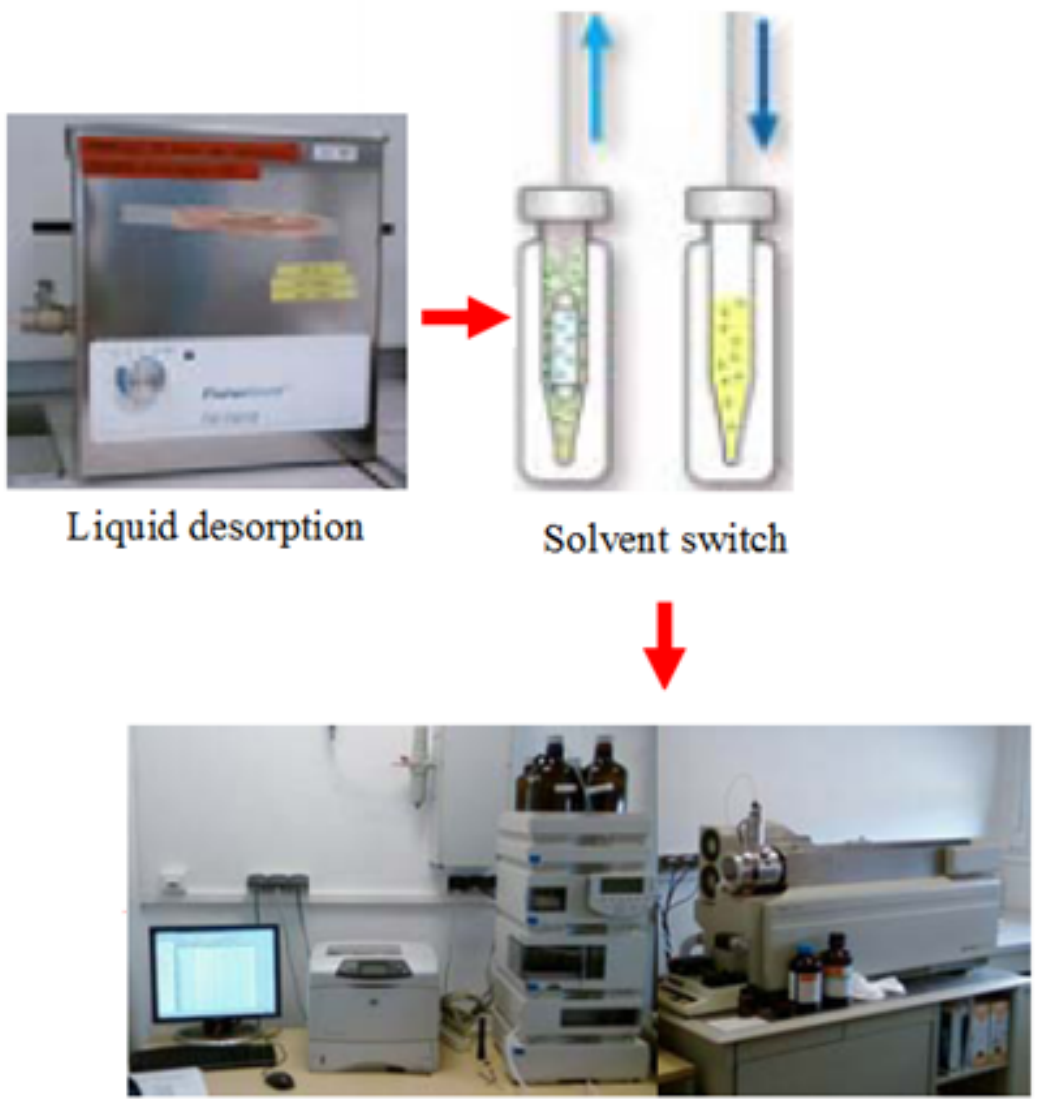

Liquid chromatography and Detector

Figure 6. Schematic representation of the SBSE liquid desorption and LC-detector instrumental set-up (Feilden, 2011; Margoum et al., 2008)

\section{Comparison of SBSE and SPME}

Although SPMS is a simple and rapid technique for sample preparation, the applicability of SPMS is occasionally limited by the small amount of coating material that is present on the fiber. In SPMS, the maximum volume of PDMS coated on the fiber is $0.5 \mu \mathrm{L}$, thereby limiting the enrichment power of the technique and resulting in low extraction efficiencies. In 1999, Baltussen and co-workers introduced SBSE to enhance the low extraction efficiencies of compounds analysed by SPME (Lokhnauth, 2005; Lanuza, 2010). It is based on the same principles as SPME, because both techniques are based on equilibrium processes but a much larger PDMS polymer is coated on a stir bar (David et al., 2003). In the SBSE technique, 55-219 $\mu$ L PDMS coatings have been used (Lokhnauth, 2005), which is about 100-300 times more than that of in SPME (Pettersson, 2004). This result an increased sensitivity factor of 100 to 1000 compared to SPMS (Lokhnauth, 2005). Since a larger volume of sorbent has been used, a greater amount of analyte will be extracted and a higher extraction efficiency (Lanuza, 2010). However, since larger volumes of PDMS are used in SBSE than in SPME, more time is required to reach equilibrium because more analyte mass will be transferred to the PDMS sorbent phase (Mitra, 2003) and the desorption process is slower than that for a SPME fiber, and thus desorption combined with cold trapping and re-concentration is 
required (David et al., 2003).

As mentioned above, the maximum volume of stationary phase coated onto an SPME fiber is ca. $0.5 \mu \mathrm{L}$. This implies that for a sample volume of $10 \mathrm{~mL}$, the phase ratio equals $2 \times 10^{4}$ and therefore quantitative extraction can only be obtained for compounds with a $K_{O / W}$ higher than $10^{5}$ (Tredoux, 2008). Since only a very limited number of compounds exhibit such high $K_{O / W}$ values, quantitative extraction unlikely occur in SPME. In SBSE on other hand, the situation is much more favorable to achieve quantitative extraction. A stir bar coated with $100 \mu \mathrm{L}$ of PDMS, used to extract $10 \mathrm{~mL}$ sample of water exhibits a $\beta$ of 100 , which implies that analytes with a $K_{O / W}$ in excess of 500 can be extracted quantitatively by SBSE. This ensures a significant increasing of sensitivity for those analytes with a $K_{O / W}$ below $10^{5}$ (Lokhnauth, 2005). Figure 7 shows that the theoretical recovery of analytes in SBSE and SPME from $10 \mathrm{~mL}$ water sample as a function of $K_{O / W}$.

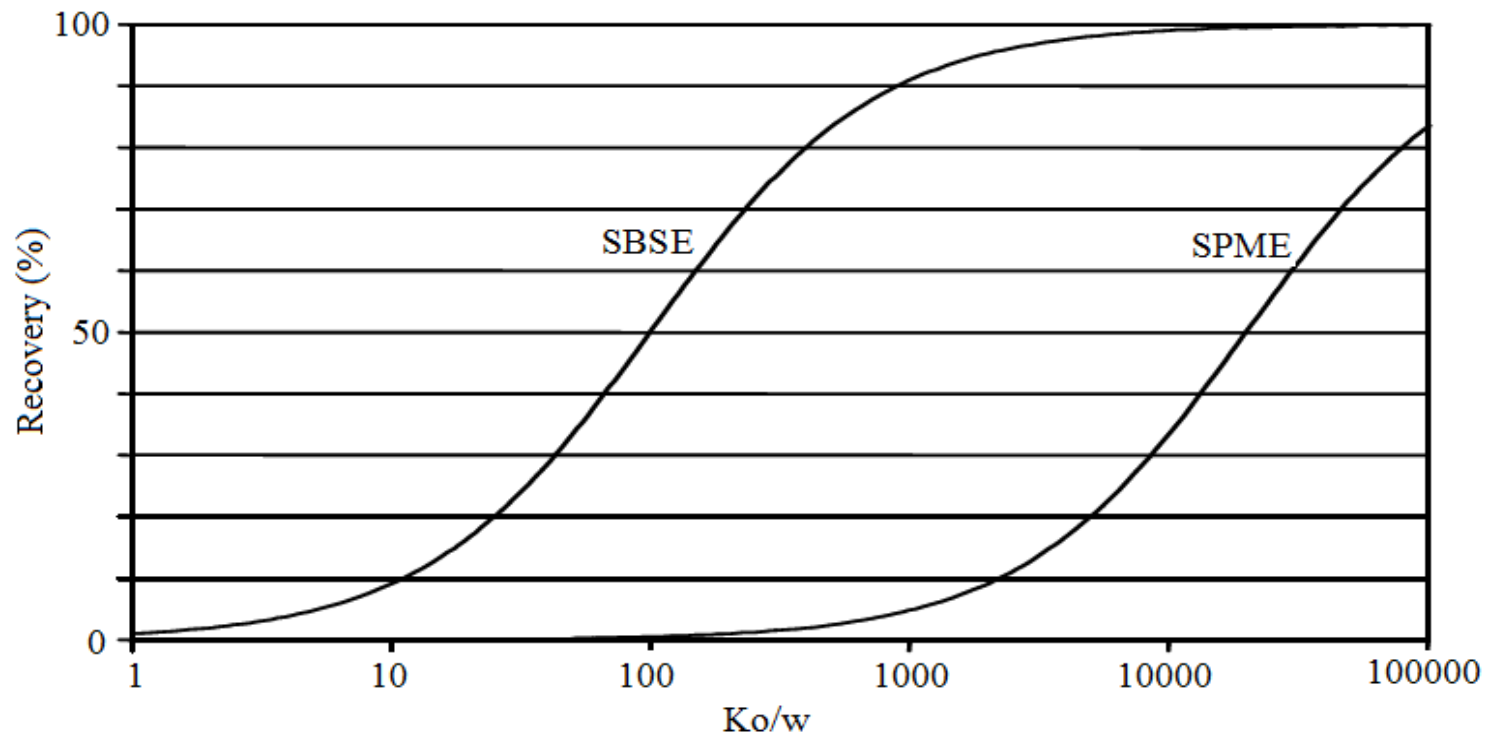

Figure 7. Theoretical recovery of SBSE and SPME as a function of $K_{O / W}$ of analytes (Tredoux, 2008)

\section{Advantages and Limitations of SBSE}

The main advantages of SBSE derive from the fact that this technique is simple to apply; solvent-free extraction technique provides quantitative analysis with extremely low detection limits (sub ppb) or highly sensitive, has excellent bar-to-bar reproducibility and is inexpensive, every bar can be re-used many times and repeatable (Corderoet al., 2009).

On the other hand, it has also some limitations or drawbacks. One of the drawbacks is related to the fact that the coated stir bar cannot be directly desorbed in a simple split/splitless injection port of a GC. Hence first the analyte has to be introduced in a TDU, which adds an additional step to the overall analytical method (Tan and Chai, 2011). For less volatile compounds, the twister can be solvent back-extracted and injected into an LC or LCMS, but the efficiency of this step is typically far less than the thermal desorption approach (Feilden, 2011). Also to be kept in mind is that the high sensitivity SBSE offers may not always be an advantage when extracting compounds present in higher concentrations as this often results in column overload. Furthermore, SBSE requires thermal desorption instrumentation, and as yet cannot be fully automated (Tredoux, 2008) because the tray transfers each desorption tube to the TDU by mechanical means (Stopforth, 2007) and removing the stir bar from the sample and drying the stir bar are performed manually, which can introduce errors. Automation of these steps is possible but this increases the cost and complexity of the hardware involved (Tan and Chai, 2011). Therefore, the cost of full instrumentation is high (Lanuza, 2010). The extraction procedure also is time-consuming (Pettersson, 2004).

However, the most important limitations of SBSE are related to the coating of stir bars. The non- polar PDMS is at present the only polymer commercially available as a coating for stir-bars (Tan and Chai, 2011). Because of the non-polar character of PDMS, SBSE is mainly applied to extract non-polar and weakly polar compounds, and fails in the extraction of strongly polar compounds (Huang et al., 2009). It only can be applied to medium-high volatility and medium-high thermally stabile analytes if a thermal desorption is to be employed, and only a few solvents compatible with PDMS can be adopted for analyte liquid desorption, sampling times are long when larger volumes of PDMS are used (Corderoet al., 2009). 


\section{Applications of SBSE}

SBSE has been successfully applied in environmental, food and biological samples (Lokhnauth, 2005).

\subsection{Environmental Analysis}

SBSE has been applied successfully in environmental analysis such as water and soil. This application includes volatile aromatics, halogenated solvents, polycyclic aromatic hydrocarbons (PAHs), organochlorine pesticide, acidic pharmaceuticals and organophosphorus pesticides and insecticides (Farajzadeh et al., 2010). Semiautomated stir bar sorptive extraction in combination with HPLC-fluorescence detection is used for the determination of PAHs in water (Hauser et al., 2002).

\subsection{Food Analysis}

Another successful application area of SBSE is the determination of minor food ingredients and food contaminants. This technique has been used to extract analytes from non-alcoholic and alcoholic beverages, dairy products, flavour and offflavour compounds in different food matrices (David et al., 2003). It has also a great application in determination of organic compounds in fruits and vegetables samples (Lokhnauth, 2005). The combination of SBSE-GC-MS with chemometrics is used for the analysis of a variety of volatile compounds in a red wine sample. This method is characterized by high sensitivity due to the large amount of sorptive phase used for SBSE, repeatability and robustness (Tredoux, 2008)

\subsection{Biological Fluids Analysis}

SBSE can also be applied to the determination of organic compounds in biological fluids (David et al., 2003). Various substances have been extracted from serum, plasma or urine. Analytes that have been successfully extracted from these matrices include phenols, steroids, fatty acids and drugs of abuse. The determination of polynuclear aromatic hydrocarbons in urine and the polychlorinated biphenyls in sperm are other applications of SBSE (Lokhnauth, 2005). SBSE technique has many practical advantages, including a small sample volume (1 $\mathrm{mL}$ ) requirement, simplicity of extraction, solvent-free and high sensitivity (Kawaguchi et al., 2008).

\section{Conclusion}

Stir bar sorptive extraction is a solventless extraction and concentration technique that can be used successfully to determine low traces of organic compounds in aqueous matrices, including water samples, biological fluids and food samples. Due to the high amount of PDMS coated on the stir bar, high sensitivity can be attained for the preconcentration of a wide range of compounds. As well-known limitation of this technique is the fact that only one sorbent (PDMS) is commercially available until this review was written. This limits the application of SBSE technique to the analysis of non-polar and some intermediate polarity compounds. Considering its applications in environmental analysis, biological analysis and food analysis, if it is joined with developments of new sorbents, interfaces, and analytical approaches, it can be concluded that SBSE certainly will occupy an important role as a major sample preparation micro technique in the near future.

\section{References}

Baltussen, H.A. (2000): New concepts in sorption based sample preparation for chromatography. PhD Thesis, Eindhoven University of Technology, Eindhoven, Netherlands.

Cordero, C., Sgorbini, B., Liberto, E., Bicchi, C., Rubiolo, P. (2009): Stir Bar Sorptive Extraction (SBSE) and Headspace Sorptive Extraction (HSSE): An Overview. LCGC North America, 27(5):376-390.

David, F., Tienpont, B., Sandra, P. (2003): Stir-Bar Sorptive extraction of trace organic compounds from aqueous matrices. LCGC North America, 21:108-118.

Farajzadeh, M.A., Djozan, D., Nouri, N., Bamorowat, M., Shalamzari, M.S. (2010): Coupling stir bar sorptive extraction dispersive liquid-liquid microextraction for preconcentration of triazole pesticides from aqueous samples followed by GC-FID and GC-MS determinations. Journal of Separation Science, 33: 1-13.

Feilden, A. (2011): Update on Undertaking Extractable and Leachable Testing. $1^{\text {st }}$ Edn, $i$ Smithers Rapra Publishing, UK, 69-84.

Hauser, B., Popp, P., Bauer, C., Kleine-Benne, E. (2002): Semi-automated stir bar sorptive extraction in combination with HPLC-fluorescence detection for the determination of polycyclic aromatic hydrocarbons in water.

http://www.gerstel.de/pdf/p-lc-an-2002-01.pdf (accessed on Oct 09, 2020)

Hoffmann, A. and Bremer, R. (1994): Design, performance and applicability of a multi-functional thermodesorption system for trace analysis in capillary GC. http://www.gerstel.cn/cn/pdf/p-gc-an-1994-01ar.pdf (accessed on Oct 09, 2020)

Huang, X., Qiu, N., Yuan, D., Lin, Q. (2009): Preparation of a mixed stir bar for sorptive extraction based on monolithic material for the extraction of quinolones from wastewater. Journal of Chromatography A. 
1217(16):2667-2673.

Jochmann, M.A. (2006): Solventless extraction and enrichment methods for compound-specific isotope analysis. $\mathrm{PhD}$ Thesis, Eberhard Karls University, Tübingen, Germany.

Kassem, M.G. (2011): Stir bar sorptive extraction for central nervous system drugs from biological fluids. Arabian Journal of Chemistry, 4(1):25-35.

Kawaguchi, M., Ito, R., Honda, H., Endo, N., Okanouchi, N., Saito, K., Seto, Y., Nakazawa, H. (2008): Measurement of benzophenones in human urine samples by stir bar sorptive extraction and thermal desorption-gas mass spectrometry. Anal Sci., 24 (11): 1509-1512.

Kole, P.L., Venkatesh, G., Kotecha, J., Sheshala, R. (2011): Recent advances in sample preparation techniques for effective bioanalytical methods. Biomed Chromatogr, 25(12):199-217

Lanuza, M.P. (2010): Pharmaceuticals And Personal care products in Environmental Waters. PhD Thesis, Rovirai Virgili University, Tarragona, Spain.

Lokhnauth, J.K. (2005): Solid phase microextraction and stir bar sorptive extraction coupled to ion mobility spectroscopy. PhD Thesis, Seton Hall University, South Orange, USA.

Margoum, C., Yang, X., Guillemain, C., Coquery, M. (2008): Stir bar sorptive extraction coupled with liquid chromatography-mass spectrometry for the quantification of pesticides in water samples.

Mitra, S. (2003): Sample preparation techniques in analytical chemistry, Vol. 162. John Wiley \& Sons, Inc., Hoboken, New Jersey, Newark, 125-138.

Ochiai, N., Sasamoto, K., Kanda, H., Pfannkoch, E. (2008): Sequential stir bar sorptive extraction for uniform enrichment of trace amounts of organic pollutants in water samples. J Chromatogr A, 1200(1):72-9.

Pettersson, J. (2004): New concepts and techniques for quantification and trace analysis by gas chromatography. PhD Thesis, Royal Institute of Technology, Stockholm, Sweden.

Skogerson, K., Wohlgemuth, G., Barupal, D.K., Fiehn, O. (2011): The volatile compound bin base mass spectral database. BMC Bioinformatics, 12(1): 321

Stopforth, A. (2007): Stir bar sorptive extraction and gas chromatography-mass spectrometry for the analysis of biological matrices. PhD Thesis, University of Stellenbosch, South Africa.

http://people.unica.it/mgpr/files/2009/01/margoum.pdf (accessed on Oct 09, 2020)

Tan, G.H. and Chai, M.K. (2011): Sample preparation in the analysis of pesticides residue in food by chromatographic techniques, pesticides.

https://cdn.intechopen.com/pdfs/12948.pdf (accessed on Oct 08, 2020)

Tredoux, A.G.J. (2008): Stir Bar Sorptive Extraction for the analysis of Beverages and Foodstuffs. PhD Thesis, University of Stellenbosch, South Africa. 